MATHEMATICS OF COMPUTATION

Volume 75, Number 255, July 2006, Pages 1175-1199

S 0025-5718(06)01834-5

Article electronically published on March 3, 2006

\title{
THE BOUNDARIES OF THE SOLUTIONS OF THE LINEAR VOLTERRA INTEGRAL EQUATIONS WITH CONVOLUTION KERNEL
}

\author{
ISMET ÖZDEMIR AND Ö. FARUK TEMIZER
}

\begin{abstract}
Some boundaries about the solution of the linear Volterra integral equations of the second type with unit source term and positive monotonically increasing convolution kernel were obtained in Ling, 1978 and 1982. A method enabling the expansion of the boundary of the solution function of an equation in this type was developed in I. Özdemir and Ö. F. Temizer, 2002.

In this paper, by using the method in Ozdemir and Temizer, it is shown that the boundary of the solution function of an equation in the same form can also be expanded under different conditions than those that they used.
\end{abstract}

\section{INTRODUCTION}

An integral equation of the form

$$
f(t)=\phi(t)-\int_{0}^{t} K(t-\tau) f(\tau) d \tau=\phi(t)-K * f
$$

is known as the second type linear Volterra integral equation with convolution kernel, where $\phi$ is the source term, $K$ is the kernel which are the known functions, and $f$ is an unknown function.

The way of obtaining a new equation equivalent to the equation of the form (1.1) is given by Theorem A, called the Equivalence Theorem by R. Ling, below.

Theorem A (Equivalence Theorem) ([2, Theorem 1.1.1]). If

(1) $K \in C^{1}[0, \infty)$,

(2) $\phi$ is locally integrable,

then the following two integral equations are equivalent:

$$
\begin{aligned}
& f(t)=\phi(t)-\int_{0}^{t} K(t-\tau) f(\tau) d \tau, \\
& f(t)=\psi(t)-\int_{0}^{t} L(t-\tau) f(\tau) d \tau,
\end{aligned}
$$

Received by the editor June 17, 2004.

2000 Mathematics Subject Classification. Primary 45D05; Secondary 45E10.

Key words and phrases. Linear Volterra integral equations with convolution kernel, equivalence theorem, convolution theorem.

(C)2006 American Mathematical Society Reverts to public domain 28 years from publication 
where

$$
\begin{gathered}
\psi(t)=\phi(t)+\int_{0}^{t} g^{\prime}(t-\tau) \phi(\tau) d \tau, \\
L(t)=g^{\prime}(t)+a g(t)+\int_{0}^{t} g(t-\tau) K^{\prime}(\tau) d \tau,
\end{gathered}
$$

and where $a=K(0), g$ is any function such that $g \in C^{1}[0, \infty)$ and $g(0)=1$.

Sufficient conditions providing the obtaining of the solution of equation (1.1) by means of $g(t)$, which is the solution of the equation

$$
g(t)=1-\int_{0}^{t} K(t-\tau) g(\tau) d \tau=1-K * g
$$

is given by Theorem $\mathrm{B}$, below:

Theorem B (Convolution Theorem) ([1, pp. 229-230]). If the conditions

$$
\begin{aligned}
& \text { (1) } \phi^{\prime}(t) \text { exists for } 0 \leq t \leq T, \quad \int_{0}^{T}\left|\phi^{\prime}(t)\right| d t<\infty(T>0), \\
& \text { (2) } \int_{0}^{T}|K(t)| d t<\infty
\end{aligned}
$$

hold, then the solution of equation (1.1) is given by

$$
f(t)=g(t) \phi(0)+\int_{0}^{t} g(t-\tau) \phi^{\prime}(\tau) d \tau=g(t) \phi(0)+g * \phi^{\prime} \quad(0 \leq t \leq T),
$$

where $g(t)$ is the solution of (1.2).

Therefore, if $g$ is known, so is $f$, or if the properties of $g$ are known, then we may be able to obtain certain properties of $f$ by (1.3).

Some boundaries about the function $f$ which is the solution of linear Volterra integral equation of the second type with unit source term and monotonically increasing kernel are obtained in Theorem $\mathrm{C}$ and Theorems 1-4, below:

Theorem C ([3, Theorem B]). Let us consider the equation

$$
f(t)=1-\int_{0}^{t} K(t-\tau) f(\tau) d \tau=1-K * f .
$$

If the conditions

$$
\begin{aligned}
& \text { (1) } K(t)>0, K^{\prime}(t)>0 \text { and } K^{\prime \prime}(t) \leq 0 \text { for } 0 \leq t<\infty \text {, } \\
& \text { (2) } 4 b \leq a^{2}
\end{aligned}
$$

hold, then the solution of (1.4) satisfies the inequality $|f(t)| \leq 1$ for $0 \leq t<\infty$, where $a=K(0)$ and $b=K^{\prime}(0)$.

Theorem 1 ([3, Theorem 3.1]). Let us consider the equation

$$
f_{1}(t)=1-\int_{0}^{t} K_{1}(t-\tau) f_{1}(\tau) d \tau=1-K_{1} * f_{1} .
$$


If

(1) $K_{1}(t)>0, K_{1}^{\prime}(t)>0, K_{1}^{\prime \prime}(t)>0$ and $K_{1}^{\prime \prime \prime}(t) \leq 0$ for $0 \leq t<\infty$,

(2) $a_{10}^{2}<4 a_{11}$

(3) $3 a_{11} \leq a_{10}^{2}$

(4) $2 a_{10}^{3}-9 a_{10} a_{11}+27 a_{12} \leq 0$,

then the solution of (1.5) satisfies the inequality $\left|f_{1}(t)\right| \leq 2$ for $0 \leq t<\infty$, where $a_{10}=K_{1}(0), a_{11}=K_{1}^{\prime}(0)$ and $a_{12}=K_{1}^{\prime \prime}(0)$.

Theorem 2 ([3, Theorem 3.3]). Let us consider the equation

$$
f_{2}(t)=1-\int_{0}^{t} K_{2}(t-\tau) f_{2}(\tau) d \tau=1-K_{2} * f_{2} .
$$

If

(1) $K_{2}(t)>0, K_{2}^{\prime}(t)>0, K_{2}^{\prime \prime}(t)>0, K_{2}^{\prime \prime \prime}(t)>0$ and $K_{2}^{(4)}(t) \leq 0$ for $0 \leq t<\infty$,

(2) $a_{20}^{2}<3 a_{21}$

(3) $\frac{8}{3} a_{21} \leq a_{20}^{2}$

(4) $a_{20}^{3}-4 a_{20} a_{21}+8 a_{22} \leq 0$,

(5) $-3 a_{20}^{4}+16 a_{20}^{2} a_{21}-64 a_{20} a_{22}+256 a_{23} \leq 0$,

then the solution of (1.6) satisfies the inequality $\left|f_{2}(t)\right| \leq 4$ for $0 \leq t<\infty$, where $a_{20}=K_{2}(0), a_{21}=K_{2}^{\prime}(0), a_{22}=K_{2}^{\prime \prime}(0)$ and $a_{23}=K_{2}^{\prime \prime \prime}(0)$.

Theorem 3. Let us consider the equation

$$
f_{3}(t)=1-\int_{0}^{t} K_{3}(t-\tau) f_{3}(\tau) d \tau=1-K_{3} * f_{3} .
$$

If

(1) $K_{3}(t)>0, K_{3}^{\prime}(t)>0, K_{3}^{\prime \prime}(t)>0, K_{3}^{\prime \prime \prime}(t)>0, K_{3}^{(4)}(t)>0$ and $K_{3}^{(5)}(t) \leq 0$ for $0 \leq t<\infty$,

(2) $a_{30}^{2}<\frac{8}{3} a_{31}$

(3) $\frac{5}{2} a_{31} \leq a_{30}^{2}$,

(4) $4 a_{30}^{3}-15 a_{30} a_{31}+25 a_{32} \leq 0$,

(5) $-3 a_{30}^{4}+15 a_{30}^{2} a_{31}-50 a_{30} a_{32}+125 a_{33} \leq 0$,

(6) $4 a_{30}^{3}-25 a_{30} a_{31}+125 a_{32}>0$,

(7) $4 a_{30}^{5}-25 a_{30}^{3} a_{31}+125 a_{30}^{2} a_{32}-625 a_{30} a_{33}+3125 a_{34} \leq 0$,

then $\left|f_{3}(t)\right| \leq 8$ for $0 \leq t<\infty$, where $a_{30}=K_{3}(0), a_{31}=K_{3}^{\prime}(0), a_{32}=K_{3}^{\prime \prime}(0)$, $a_{33}=K_{3}^{\prime \prime \prime}(0)$ and $a_{34}=K_{3}^{(4)}(0)$, (for the proof, see [4]).

Theorem 4. Let us consider the equation

$$
f_{4}(t)=1-\int_{0}^{t} K_{4}(t-\tau) f_{4}(\tau) d \tau=1-K_{4} * f_{4}
$$


(1) $K_{4}(t)>0, K_{4}^{\prime}(t)>0, K_{4}^{\prime \prime}(t)>0, K_{4}^{\prime \prime \prime}(t)>0, K_{4}^{(4)}(t)>0, K_{4}^{(5)}(t)>0$

$$
\text { and } K_{4}^{(6)}(t) \leq 0 \text { for } 0 \leq t<\infty
$$

(2) $a_{40}^{2}<\frac{5}{2} a_{41}$

(3) $\frac{12}{5} a_{41} \leq a_{40}^{2}$

(4) $5 a_{40}^{3}-18 a_{40} a_{41}+27 a_{42} \leq 0$,

(5) $-5 a_{40}^{4}+24_{40}^{2} a_{41}-72 a_{40} a_{42}+144 a_{43} \leq 0$,

(6) $7 a_{40}^{3}-36 a_{40} a_{41}+108 a_{42}>0$,

(7) $\quad a_{40}^{5}-6 a_{40}^{3} a_{41}+27 a_{40}^{2} a_{42}-108 a_{40} a_{43}+324 a_{44} \leq 0$,

(8) $5 a_{40}^{3}-36 a_{40} a_{41}+216 a_{42}>0$,

(9) $-5 a_{40}^{4}+36 a_{40}^{2} a_{41}-216 a_{40} a_{42}+1296 a_{43}>0$,

(10) $-5 a_{40}^{6}+36 a_{40}^{4} a_{41}-216 a_{40}^{3} a_{42}+1296 a_{40}^{2} a_{43}-7776 a_{40} a_{44}+46656 a_{45} \leq 0$,

then $\left|f_{4}(t)\right| \leq 16$ for $0 \leq t<\infty$, where $a_{40}=K_{4}(0), a_{41}=K_{4}^{\prime}(0), a_{42}=K_{4}^{\prime \prime}(0)$, $a_{43}=K_{4}^{\prime \prime \prime}(0), a_{44}=K_{4}^{(4)}(0)$ and $a_{45}=K_{4}^{(5)}(0)$, (for the proof, see [4]).

In the proof of Theorem 1, the equivalent of (1.5) is found by first using the Equivalence Theorem, and second it is obtained that the kernel of the new equation with unit source term related to equivalent equation satisfies the conditions of Theorem C. Thus, by using Theorems C and B, respectively, a boundary $\left|f_{1}(t)\right| \leq 2$ for the function $f_{1}$, which is the solution of equation (1.5), is obtained [3].

Also, in the proof of Theorem 2, first, the relation which is equivalent to (1.6) is found by using the Equivalence Theorem, and second, it is obtained that the kernel of the new equation with unit source term related to equivalent equation satisfies the conditions of Theorem 1, $[3$. Thus, by using Theorems 1 and B, respectively, a boundary $\left|f_{2}(t)\right| \leq 4$ is found for the function $f_{2}$, which is the solution of equation (1.6).

By the same method, in Theorem 3, by using Theorems A, 2 and B, respectively, it is obtained that a boundary is $\left|f_{3}(t)\right| \leq 8$ for the function $f_{3}$, which is the solution of equation (1.7), 4].

Also, by using Theorems A, 3 and B, respectively, a boundary $\left|f_{4}(t)\right| \leq 16$ is obtained for the function $f_{4}$, which is the solution of equation (1.8), 4, Theorem 4].

Furthermore, by using the method used in the proof of Theorems 1-4 successively, it is concluded that a boundary $\left|f_{n}(t)\right| \leq 2^{n}$ for the function $f_{n}$, which is the solution of the equation

$$
f_{n}(t)=1-\int_{0}^{t} K_{n}(t-\tau) f_{n}(\tau) d \tau=1-K_{n} * f_{n}(\mathrm{n} \text { is an integer greater than } 1)
$$

with unit source term and monotonically increasing convolution kernel, could be obtained, 4]. The sufficient conditions concerning obtaining a solution of equation (1.9), as above, are given by Theorem $2 . n$ which is the generalization of Theorem 2. 


\section{The MAIN RESUltS}

In the case $a_{10}^{2} \geq 4 a_{11}$ of Theorem 1 , the new conditions enabling the validity of the consequence of Theorem 1 are given by Theorem 2.1, below, which is different than Theorem 1 of R. Ling [3].

In the present paper, it is first obtained, in the proof of Theorem 2.2 that equation (2.5) is equivalent to equation (2.4) by the Equivalence Theorem. Besides, it is obtained that the kernel $L_{12}$ of equation (2.7) satisfies the conditions of Theorem 2.1. Thus, by using Theorems 2.1 and B, respectively, a boundary $\left|f_{12}(t)\right| \leq 4$ is obtained for the function $f_{12}$, which is the solution of equation (2.4).

Also, in the proof of Theorem 2.3, first, 2.15), which is equivalent to (2.14) is found by the aid of the Equivalence Theorem, and later it is obtained that the kernel $L_{13}$ of equation (2.17) satisfies the conditions of Theorem 2.2. Thus, by using Theorems 2.2 and $\mathrm{B}$, respectively, a boundary $\left|f_{13}(t)\right| \leq 8$ is found for the function $f_{13}$ which is the solution of equation (2.14).

Besides, in the proof of Theorem 2.4, first, (2.25), which is equivalent to (2.24), is found by using the Equivalence Theorem, and later it is obtained that the kernel $L_{14}$ of equation (2.27) satisfies the conditions of Theorem 2.3. Thus, by using Theorems 2.3 and B, respectively, a boundary $\left|f_{14}(t)\right| \leq 16$ is found for the function $f_{14}$ which is the solution of equation (2.24).

Furthermore, by employing the method used in the proofs of Theorems $2.2-2.4$, successively, it is concluded that a boundary $\left|f_{1 n}(t)\right| \leq 2^{n}$, for the solution function $f_{1 n}$ of any equation of the form

$$
f_{1 n}(t)=1-\int_{0}^{t} K_{1 n}(t-\tau) f_{1 n}(\tau) d \tau=1-K_{1 n} * f_{1 n}
$$

with unit source term and monotonically increasing convolution kernel, has been obtained in Theorem 2.n which is the generalization of Theorem 2.1.

Also, the infinitely many numbers of kernels $K_{1 n}$ of the form

$$
K_{1 n}(t)=\sum_{m=0}^{n+1} c_{m} t^{n+1-m}+c_{n+2} e^{-t}
$$

satisfying the conditions of Theorem 2.n are derived by a method.

Hereafter, we assume unless stated otherwise that $t \in[0, \infty)$ and $n$ is an arbitrary element of $\mathbb{N}=\{1,2,3, \ldots\}$.

Theorem 2.1 ([3, Theorem 3.2]). Let us consider the equation of the form

$$
f_{11}(t)=1-\int_{0}^{t} K_{11}(t-\tau) f_{11}(\tau) d \tau=1-K_{11} * f_{11} .
$$

Suppose that the conditions

(1) $K_{11}(t)>0, K_{11}^{\prime}(t)>0, K_{11}^{\prime \prime}(t)>0$ and $K_{11}^{\prime \prime \prime}(t) \leq 0$ for $0 \leq t<\infty$,

(2) $a_{110}^{2}<\frac{9}{2} a_{111}$

(3) $4 a_{111} \leq a_{110}^{2}$,

(4) $2 a_{110}^{3}-9 a_{110} a_{111}+27 a_{112} \leq 0$,

hold. Then the solution of (2.1) satisfies the inequality $\left|f_{11}(t)\right| \leq 2$ for $0 \leq t<\infty$, where $a_{110}=K_{11}(0), a_{111}=K_{11}^{\prime}(0), a_{112}=K_{11}^{\prime \prime}(0)$. 
We should note that condition (2) of Theorem 2.1 is equivalent to the inequality given [3]:

$$
\frac{a_{110}}{3}<\frac{a_{110}-\sqrt{a_{110}^{2}-4 a_{111}}}{2} .
$$

Now, we can give a function $K_{11}$ satisfying the conditions of Theorem 2.1, as follows:

Example 2.1. If there exist the numbers $a_{110}, a_{111}, a_{112}>0$ satisfying conditions (2)-(4) of Theorem 2.1, then there exists at least one function $K_{11}$ which satisfies condition (1) of Theorem 2.1, of the form

$$
K_{11}(t)=\sum_{m=0}^{2} c_{m} t^{2-m}+c_{3} e^{-t}
$$

such that

$$
K_{11}(0)=a_{110}, K_{11}^{\prime}(0)=a_{111}, K_{11}^{\prime \prime}(0)=a_{112} .
$$

To see the validity of this assertion, we must first show that there exist the numbers $a_{110}, a_{111}, a_{112}>0$ satisfying conditions (2)-(4) of Theorem 2.1. Let us choose the numbers $a_{110}$ and $a_{111}$ satisfying conditions (2) and (3) of Theorem 2.1 and define $P_{11 i}$ for all $i \in \mathbb{N}_{3}$ and $\gamma_{11}$ by

$$
P_{11 i}(\gamma)=\sum_{k=0}^{i}(-\gamma)^{i-k} a_{11(k-1)}\left(a_{11(-1)} \equiv 1, \quad \text { by convention }\right) \text { and } \gamma_{11}=\frac{a_{110}}{3} \text {. }
$$

By $\mathbb{N}_{k}$, hereafter we mean the set of positive integers all of which are less than or equal to $k \in \mathbb{N}$, that is, $\mathbb{N}_{k}=\{1,2,3, \ldots, k\}$.

Since condition (4) of Theorem 2.1 is equivalent to

$$
P_{113}\left(\gamma_{11}\right)=-\gamma_{11} P_{112}\left(\gamma_{11}\right)+a_{112} \leq 0,
$$

the number $a_{112}>0$ can be chosen as

$$
0<a_{112} \leq \gamma_{11} P_{112}\left(\gamma_{11}\right) .
$$

Thus, the numbers $a_{110}, a_{111}, a_{112}$ fulfill conditions (2)-(4) of Theorem 2.1.

The solution of the system of linear equations (2.3) which is equivalent to

$$
c_{2}+c_{3}=a_{110}, c_{1}-c_{3}=a_{111}, 2 c_{0}+c_{3}=a_{112}
$$

is obtained as

$$
\left(c_{0}, c_{1}, c_{2}, c_{3}\right)=\left(\frac{a_{112}-c_{3}}{2}, a_{111}+c_{3}, a_{110}-c_{3}, c_{3}\right),
$$

where $c_{3}$ is an arbitrary constant such that

$$
0 \leq c_{3}<\min \left\{a_{110}, a_{112}\right\} .
$$

Hence, every function $K_{11}$ given by (2.2) also satisfies condition (1) of Theorem 2.1 .

For example, if the numbers $a_{110}$ and $a_{111}$ are taken as $a_{110}=\frac{21}{10}$ and $a_{111}=1$, then $\gamma_{11}=\frac{7}{10}$ and the number $a_{112}$ satisfying the inequality

$$
0<a_{112} \leq \gamma_{11} P_{112}\left(\gamma_{11}\right)=\frac{14}{1000}
$$


can be chosen as $a_{112}=\frac{1}{100}$. Also, $c_{3}$ satisfying the inequality

$$
0 \leq c_{3}<\min \left\{a_{110}, a_{112}\right\}
$$

can be taken as $c_{3}=\frac{1}{200}$. Then,

$$
c_{0}=\frac{1}{400}, c_{1}=\frac{201}{200}, c_{2}=\frac{419}{200}
$$

and

$$
K_{11}(t)=\frac{1}{400} t^{2}+\frac{201}{200} t+\frac{419}{200}+\frac{1}{200} e^{-t} .
$$

Theorem 2.2. Let us consider the equation

$$
f_{12}(t)=1-\int_{0}^{t} K_{12}(t-\tau) f_{12}(\tau) d \tau=1-K_{12} * f_{12} .
$$

Suppose that the conditions

(1) $K_{12}(t)>0, K_{12}^{\prime}(t)>0, K_{12}^{\prime \prime}(t)>0, K_{12}^{\prime \prime \prime}(t)>0$ and $K_{12}^{(4)}(t) \leq 0$ for $0 \leq t<\infty$,

(2) $a_{120}^{2}<\frac{28}{9} a_{121}$,

(3) $3 a_{121} \leq a_{120}^{2}$,

(4) $106 a_{120}^{3}-405 a_{120} a_{121}+729 a_{122} \leq 0$,

(5) $-2 a_{120}^{4}+9 a_{120}^{2} a_{121}-27 a_{120} a_{122}+81 a_{123} \leq 0$,

hold. Then the solution of (2.4) satisfies the inequality $\left|f_{12}(t)\right| \leq 4$ for $0 \leq t<\infty$, where $a_{120}=K_{12}(0), a_{121}=K_{12}^{\prime}(0), a_{122}=K_{12}^{\prime \prime}(0), a_{123}=K_{12}^{\prime \prime \prime}(0)$.

Proof. If the function $g$ is taken as $g(t)=e^{-\gamma t}(\gamma \in \mathbb{R})$ in Theorem A, then it is observed that

$$
f_{12}(t)=e^{-\gamma t}-L_{12} * f_{12}
$$

which is equivalent to (2.4), where

$$
L_{12}(t)=\left(a_{120}-\gamma\right) e^{-\gamma t}+K_{12}^{\prime} * e^{-\gamma t} .
$$

By differentiating (2.6), it is obtained by $L_{12}^{\prime}(t), L_{12}^{\prime \prime}(t)$ and $L_{12}^{\prime \prime \prime}(t)$ that

$$
\begin{aligned}
& L_{12}^{\prime}(t)=\left(\gamma^{2}-a_{120} \gamma+a_{121}\right) e^{-\gamma t}+K_{12}^{\prime \prime} * e^{-\gamma t}, \\
& L_{12}^{\prime \prime}(t)=\left(-\gamma^{3}+a_{120} \gamma^{2}-a_{121} \gamma+a_{122}\right) e^{-\gamma t}+K_{12}^{\prime \prime \prime} * e^{-\gamma t}
\end{aligned}
$$

and

$$
L_{12}^{\prime \prime \prime}(t)=\left(\gamma^{4}-a_{120} \gamma^{3}+a_{121} \gamma^{2}-a_{122} \gamma+a_{123}\right) e^{-\gamma t}+K_{12}^{(4)} * e^{-\gamma t} .
$$

Now, one can see that the kernel $L_{12}$ of

$$
h_{12}(t)=1-L_{12} * h_{12}
$$

satisfies the conditions of Theorem 2.1 .

The corresponding inequalities to conditions (2) and (3) of Theorem 2.1 are

$$
\left[L_{12}(0)\right]^{2}<\frac{9}{2} L_{12}^{\prime}(0)
$$

and

$$
4 L_{12}^{\prime}(0) \leq\left[L_{12}(0)\right]^{2},
$$


respectively. Inequalities (2.8) and (2.9) are equivalent to

$$
\left(a_{120}-\gamma\right)^{2}<\frac{9}{2}\left(\gamma^{2}-a_{120} \gamma+a_{121}\right)
$$

and

$$
4\left(\gamma^{2}-a_{120} \gamma+a_{121}\right) \leq\left(a_{120}-\gamma\right)^{2},
$$

respectively. Inequality (2.10) is equivalent to

$$
q_{1}(\gamma)=7 \gamma^{2}-5 a_{120} \gamma+9 a_{121}-2 a_{120}^{2}>0 .
$$

The discriminant of $q_{1}(\gamma)=0$ is $9\left(9 a_{120}^{2}-28 a_{121}\right)$, which is negative by condition (2) of Theorem 2.2. Hence, $q_{1}(\gamma)>0$ for every $\gamma \in \mathbb{R}$.

Inequality (2.11) is equivalent to

$$
q_{2}(\gamma)=3 \gamma^{2}-2 a_{120} \gamma+4 a_{121}-a_{120}^{2} \leq 0 .
$$

The discriminant of $q_{2}(\gamma)=0$ is $16\left(a_{120}^{2}-3 a_{121}\right)$, which is nonnegative by condition (3) of Theorem 2.2. Hence, if $\gamma$ is taken as $\gamma=\gamma_{12}=\frac{a_{120}}{3}$, then $q_{2}(\gamma)=q_{2}\left(\gamma_{12}\right) \leq 0$.

The inequality corresponding to condition (4) of Theorem 2.1 is

$$
2\left[L_{12}(0)\right]^{3}-9 L_{12}(0) L_{12}^{\prime}(0)+27 L_{12}^{\prime \prime}(0) \leq 0
$$

or

$$
\begin{aligned}
q_{3}(\gamma)= & 2\left(a_{120}-\gamma\right)^{3}-9\left(a_{120}-\gamma\right)\left(\gamma^{2}-a_{120} \gamma+a_{121}\right) \\
& +27\left(-\gamma^{3}+a_{120} \gamma^{2}-a_{121} \gamma+a_{122}\right) \leq 0 .
\end{aligned}
$$

By condition (4) of Theorem 2.2,

$$
q_{3}\left(\gamma_{12}\right)=\frac{1}{27}\left(106 a_{120}^{3}-405 a_{120} a_{121}+729 a_{122}\right) \leq 0 .
$$

The corresponding inequalities to condition (1) of Theorem 2.1 are

$$
L_{12}(t), L_{12}^{\prime}(t), L_{12}^{\prime \prime}(t)>0 \text { and } L_{12}^{\prime \prime \prime}(t) \leq 0 .
$$

Since $a_{120}-\gamma_{12}$ is positive, $L_{12}(t)>0$ and the discriminant of $\gamma^{2}-a_{120} \gamma+a_{121}=0$ is negative by condition (2) of Theorem 2.2, $\gamma_{12}^{2}-a_{120} \gamma_{12}+a_{121}>0$, and so $L_{12}^{\prime}(t)>0$.

From condition (5) of Theorem 2.2,

$$
-\gamma_{12}^{3}+a_{120} \gamma_{12}^{2}-a_{121} \gamma_{12}+a_{122}=\frac{1}{27}\left(2 a_{120}^{3}-9 a_{120} a_{121}+27 a_{122}\right)>0
$$

and

$$
\begin{aligned}
\gamma_{12}^{4}- & a_{120} \gamma_{12}^{3}+a_{121} \gamma_{12}^{2}-a_{122} \gamma_{12}+a_{123} \\
& =\frac{1}{81}\left(-2 a_{120}^{4}+9 a_{120}^{2} a_{121}-27 a_{120} a_{122}+81 a_{123}\right) \leq 0 .
\end{aligned}
$$

Therefore, $L_{12}^{\prime \prime}(t)>0, L_{12}^{\prime \prime \prime}(t) \leq 0$. So, the solution of equation (2.7) satisfies the inequality $\left|h_{12}(t)\right| \leq 2$.

By Theorem B, the solution of equation (2.5) can be expressed as

$$
f_{12}(t)=h_{12}(t)-\gamma_{12} \int_{0}^{t} h_{12}(t-\tau) e^{-\gamma_{12} \tau} d \tau
$$

and thus,

$$
\left|f_{12}(t)\right| \leq 2+2 \gamma_{12} \int_{0}^{t} e^{-\gamma_{12} \tau} d \tau=2-2\left(e^{-\gamma_{12} t}-1\right) \leq 4,
$$

which completes the proof of Theorem 2.2 . 
A function $K_{12}$ satisfying conditions (1)-(5) of Theorem 2.2 can be obtained by the following method:

Example 2.2. If there exist the numbers $a_{120}, a_{121}, a_{122}, a_{123}>0$ satisfying conditions (2)-(5) of Theorem 2.2, then there exists at least one function $K_{12}$ which satisfies condition (1) of Theorem 2.2 of the form

$$
K_{12}(t)=\sum_{m=0}^{3} c_{m} t^{3-m}+c_{4} e^{-t}
$$

such that

$$
K_{12}(0)=a_{120}, K_{12}^{\prime}(0)=a_{121}, K_{12}^{\prime \prime}(0)=a_{122}, K_{12}^{\prime \prime \prime}(0)=a_{123} .
$$

First, let us see that there exist the numbers $a_{120}, a_{121}, a_{122}, a_{123}>0$ satisfying conditions (2)-(5) of Theorem 2.2. It is clear that the numbers $a_{120}$ and $a_{121}$ satisfying conditions (2) and (3) of Theorem 2.2 exist. Now, we shall define the polynomial $P_{12 i}$ for all $i \in \mathbb{N}_{4}$ and the number $\gamma_{12}$ by

$$
P_{12 i}(\gamma)=\sum_{k=0}^{i}(-\gamma)^{i-k} a_{12(k-1)}\left(a_{12(-1)} \equiv 1 \text {, by convention) and } \gamma_{12}=\frac{a_{120}}{3}\right. \text {. }
$$

Then, it can be clearly obtained by the proof of Theorem 2.2 that the numbers $a_{110}$ and $a_{111}$ satisfying conditions (2) and (3) of Theorem 2.1 can be defined by

$$
a_{110}=P_{121}\left(\gamma_{12}\right) \text { and } a_{111}=P_{122}\left(\gamma_{12}\right) \text {. }
$$

Besides, the number $a_{112}>0$ can be found by means of $a_{110}$ and $a_{111}$ as it was derived in Example 2.1. Since condition (4) of Theorem 2.2 is equivalent to

$$
2\left[P_{121}\left(\gamma_{12}\right)\right]^{3}-9 P_{121}\left(\gamma_{12}\right) P_{122}\left(\gamma_{12}\right)+27 P_{123}\left(\gamma_{12}\right) \leq 0,
$$

$a_{122}$ is found as

$$
a_{122}=a_{112}+\gamma_{12} P_{122}\left(\gamma_{12}\right)=a_{112}+\gamma_{12} a_{111}
$$

if $P_{123}\left(\gamma_{12}\right)$ is taken as

$$
P_{123}\left(\gamma_{12}\right)=-\gamma_{12} P_{122}\left(\gamma_{12}\right)+a_{122}=a_{112} .
$$

Furthermore, since condition (5) of Theorem 2.2 is equivalent to

$$
P_{124}\left(\gamma_{12}\right)=-\gamma_{12} P_{123}\left(\gamma_{12}\right)+a_{123} \leq 0,
$$

the number $a_{123}>0$ can be chosen as

$$
0<a_{123} \leq \gamma_{12} P_{123}\left(\gamma_{12}\right)=\gamma_{12} a_{112}
$$

Clearly, the numbers $a_{120}, a_{121}, a_{122}, a_{123}>0$, obtained above, satisfy conditions (2)-(5) of Theorem 2.2. The solution of the system of linear equations (2.13) which is equivalent to

$$
c_{3}+c_{4}=a_{120}, c_{2}-c_{4}=a_{121}, 2 c_{1}+c_{4}=a_{122}, 6 c_{0}-c_{4}=a_{123}
$$

is obtained as

$$
\left(c_{0}, c_{1}, c_{2}, c_{3}, c_{4}\right)=\left(\frac{a_{123}+c_{4}}{6}, \frac{a_{122}-c_{4}}{2}, a_{121}+c_{4}, a_{120}-c_{4}, c_{4}\right),
$$

where $c_{4}$ is an arbitrary constant satisfying the inequality

$$
\max \left\{-a_{121},-a_{123}\right\}<c_{4} \leq 0 .
$$


Hence, every function $K_{12}$ of the form (2.12) also satisfies condition (1) of Theorem 2.2 .

For example, if the numbers $a_{120}$ and $a_{121}$ are taken as $a_{120}=\frac{26}{5}$ and $a_{121}=$ 9 , then $\gamma_{12}=\frac{26}{15}$,

$$
a_{110}=P_{121}\left(\gamma_{12}\right)=\frac{52}{15} \text { and } a_{111}=P_{122}\left(\gamma_{12}\right)=\frac{673}{225} .
$$

By using Example 2.1, $\gamma_{11}=\frac{52}{45}$ and the number $a_{112}>0$ satisfying the inequality

$$
0<a_{112} \leq \gamma_{11} P_{112}\left(\gamma_{11}\right)=\frac{33748}{91125}
$$

can be chosen as $a_{112}=\frac{3}{10}$. Hence,

$$
a_{122}=a_{112}+\gamma_{12} a_{111}=\frac{37021}{6750} \text {. }
$$

Furthermore, the number $a_{123}>0$ satisfying the inequality

$$
0<a_{123} \leq \gamma_{12} a_{112}=\frac{13}{25}
$$

can be taken as $a_{123}=\frac{1}{2}$. Additionally, the number $c_{4}$ satisfying the inequality

$$
\max \left\{-a_{121},-a_{123}\right\}=\max \left\{-9,-\frac{1}{2}\right\}<c_{4} \leq 0
$$

can be taken as $c_{4}=-\frac{1}{3}$. Therefore,

$$
\left(c_{0}, c_{1}, c_{2}, c_{3}, c_{4}\right)=\left(\frac{1}{36}, \frac{39271}{13500}, \frac{26}{3}, \frac{83}{15},-\frac{1}{3}\right),
$$

and thus,

$$
K_{12}(t)=\frac{1}{36} t^{3}+\frac{39271}{13500} t^{2}+\frac{26}{3} t+\frac{83}{15}-\frac{1}{3} e^{-t} .
$$

Theorem 2.3. Let us consider the equation of the form

$$
f_{13}(t)=1-\int_{0}^{t} K_{13}(t-\tau) f_{13}(\tau) d \tau=1-K_{13} * f_{13}
$$

under the following assumptions:

(1) $K_{13}(t)>0, K_{13}^{\prime}(t)>0, K_{13}^{\prime \prime}(t)>0, K_{13}^{\prime \prime \prime}(t)>0, K_{13}^{(4)}(t)>0$ and $K_{13}^{(5)}(t) \leq 0$ for $0 \leq t<\infty$,

(2) $a_{130}^{2}<\frac{19}{7} a_{131}$

(3) $\frac{8}{3} a_{131} \leq a_{130}^{2}$,

(4) $161 a_{130}^{3}-576 a_{130} a_{131}+864 a_{132} \leq 0$,

(5) $-11 a_{130}^{4}+48 a_{130}^{2} a_{131}-128 a_{130} a_{132}+256 a_{133} \leq 0$,

(6) $3 a_{130}^{3}-16 a_{130} a_{131}+64 a_{132}>0$,

(7) $3 a_{130}^{5}-16 a_{130}^{3} a_{131}+64 a_{130}^{2} a_{132}-256 a_{130} a_{133}+1024 a_{134} \leq 0$,

where $a_{130}=K_{13}(0), a_{131}=K_{13}^{\prime}(0), a_{132}=K_{13}^{\prime \prime}(0), a_{133}=K_{13}^{\prime \prime \prime}(0), a_{134}=$ $K_{13}^{(4)}(0)$. Then, the solution of (2.14) satisfies the inequality $\left|f_{13}(t)\right| \leq 8$ for $0 \leq$ $t<\infty$. 
Proof. The equation (2.14) may be written in the form $f_{13}(t)=1-K_{13} * f_{13}$. By taking $g(t)=e^{-\gamma t}(\gamma \in \mathbb{R})$ in Theorem A, the equivalent equation is obtained as

$$
f_{13}(t)=e^{-\gamma t}-L_{13} * f_{13},
$$

where

$$
L_{13}(t)=\left(a_{130}-\gamma\right) e^{-\gamma t}+K_{13}^{\prime} * e^{-\gamma t} .
$$

By differentiating (2.16), it is found by $L_{13}^{\prime}(t), L_{13}^{\prime \prime}(t), L_{13}^{\prime \prime \prime}(t)$ and $L_{13}^{(4)}(t)$ that

$$
\begin{aligned}
& L_{13}^{\prime}(t)=\left(\gamma^{2}-a_{130} \gamma+a_{131}\right) e^{-\gamma t}+K_{13}^{\prime \prime} * e^{-\gamma t}, \\
& L_{13}^{\prime \prime}(t)=\left(-\gamma^{3}+a_{130} \gamma^{2}-a_{131} \gamma+a_{132}\right) e^{-\gamma t}+K_{13}^{\prime \prime \prime} * e^{-\gamma t}, \\
& L_{13}^{\prime \prime \prime}(t)=\left(\gamma^{4}-a_{130} \gamma^{3}+a_{131} \gamma^{2}-a_{132} \gamma+a_{133}\right) e^{-\gamma t}+K_{13}^{(4)} * e^{-\gamma t}
\end{aligned}
$$

and

$$
L_{13}^{(4)}(t)=\left(-\gamma^{5}+a_{130} \gamma^{4}-a_{131} \gamma^{3}+a_{132} \gamma^{2}-a_{133} \gamma+a_{134}\right) e^{-\gamma t}+K_{13}^{(5)} * e^{-\gamma t} .
$$

Let us turn to show that the kernel $L_{13}$ of the equation

$$
h_{13}(t)=1-L_{13} * h_{13}
$$

satisfies all the conditions of Theorem 2.2. The corresponding inequalities to conditions (2) and (3) of Theorem 2.2 are

$$
\left[L_{13}(0)\right]^{2}<\frac{28}{9} L_{13}^{\prime}(0)
$$

and

$$
3 L_{13}^{\prime}(0) \leq\left[L_{13}(0)\right]^{2},
$$

respectively. Inequalities (2.18) and (2.19) are equivalent to

$$
\left(a_{130}-\gamma\right)^{2}<\frac{28}{9}\left(\gamma^{2}-a_{130} \gamma+a_{131}\right)
$$

and

$$
3\left(\gamma^{2}-a_{130} \gamma+a_{131}\right) \leq\left(a_{130}-\gamma\right)^{2} .
$$

Since inequality (2.20) is equivalent to

$$
q_{1}(\gamma)=19 \gamma^{2}-10 a_{130} \gamma+28 a_{131}-9 a_{130}^{2}>0
$$

and the discriminant of $q_{1}(\gamma)=0$ is $112\left(7 a_{130}^{2}-19 a_{131}\right)$, which is negative by condition (2) of Theorem 2.3, $q_{1}(\gamma)>0$ for every $\gamma \in \mathbb{R}$.

Since inequality (2.21) is equivalent to

$$
q_{2}(\gamma)=2 \gamma^{2}-a_{130} \gamma+3 a_{131}-a_{130}^{2} \leq 0
$$

and the discriminant of $q_{2}(\gamma)=0$ is $3\left(3 a_{130}^{2}-8 a_{131}\right)$, which is nonnegative by condition (3) of Theorem 2.3, if $\gamma$ is chosen as $\gamma=\gamma_{13}=\frac{a_{130}}{4}$, then $q_{2}\left(\gamma_{13}\right) \leq 0$.

The inequality corresponding to condition (4) of Theorem 2.2 is

$$
106\left[L_{13}(0)\right]^{3}-405 L_{13}(0) L_{13}^{\prime}(0)+729 L_{13}^{\prime \prime}(0) \leq 0,
$$

which is equivalent to

$$
\begin{aligned}
q_{3}(\gamma)= & 106\left(a_{130}-\gamma\right)^{3}-405\left(a_{130}-\gamma\right)\left(\gamma^{2}-a_{130} \gamma+a_{131}\right) \\
& +729\left(-\gamma^{3}+a_{130} \gamma^{2}-a_{131} \gamma+a_{132}\right) \leq 0 .
\end{aligned}
$$


By condition (4) of Theorem 2.3,

$$
q_{3}\left(\gamma_{13}\right)=\frac{27}{32}\left(161 a_{130}^{3}-576 a_{130} a_{131}+864 a_{132}\right) \leq 0 .
$$

The inequality corresponding to condition (5) of Theorem 2.2 is

$$
-2\left[L_{13}(0)\right]^{4}+9\left[L_{13}(0)\right]^{2} L_{13}^{\prime}(0)-27 L_{13}(0) L_{13}^{\prime \prime}(0)+81 L_{13}^{\prime \prime \prime}(0) \leq 0,
$$

which is equivalent to

$$
\begin{aligned}
q_{4}(\gamma)= & -2\left(a_{130}-\gamma\right)^{4}+9\left(a_{130}-\gamma\right)^{2}\left(\gamma^{2}-a_{130} \gamma+a_{131}\right) \\
& -27\left(a_{130}-\gamma\right)\left(-\gamma^{3}+a_{130} \gamma^{2}-a_{131} \gamma+a_{132}\right)+ \\
& +81\left(\gamma^{4}-a_{130} \gamma^{3}+a_{131} \gamma^{2}-a_{132} \gamma+a_{133}\right) \leq 0 .
\end{aligned}
$$

By condition (5) of Theorem 2.3,

$$
q_{4}\left(\gamma_{13}\right)=\frac{81}{256}\left(-11 a_{130}^{4}+48 a_{130}^{2} a_{131}-128 a_{130} a_{132}+256 a_{133}\right) \leq 0 .
$$

Furthermore, $a_{130}-\gamma_{13}$ is positive. Thus, $L_{13}(t)>0$. Since the discriminant of $\gamma^{2}-a_{130} \gamma+a_{131}=0$ is negative by condition (2) of Theorem 2.3, $\gamma_{13}^{2}-a_{130} \gamma_{13}+$ $a_{131}>0$ and so, $L_{13}^{\prime}(t)>0$. Additionally, by condition (6) of Theorem 2.3,

$$
-\gamma_{13}^{3}+a_{130} \gamma_{13}^{2}-a_{131} \gamma_{13}+a_{132}=\frac{1}{64}\left(3 a_{130}^{3}-16 a_{130} a_{131}+64 a_{132}\right)>0
$$

and thus, $L_{13}^{\prime \prime}(t)>0$. By condition (7) of Theorem 2.3,

$$
\begin{aligned}
\gamma_{13}^{4}- & a_{130} \gamma_{13}^{3}+a_{131} \gamma_{13}^{2}-a_{132} \gamma_{13}+a_{133} \\
& =\frac{1}{256}\left(-3 a_{130}^{4}+16 a_{130}^{2} a_{131}-64 a_{130} a_{132}+256 a_{133}\right)>0
\end{aligned}
$$

and

$$
\begin{aligned}
-\gamma_{13}^{5} & +a_{130} \gamma_{13}^{4}-a_{131} \gamma_{13}^{3}+a_{132} \gamma_{13}^{2}-a_{133} \gamma_{13}+a_{134} \\
= & \frac{1}{1024}\left(3 a_{130}^{5}-16 a_{130}^{3} a_{131}+64 a_{130}^{2} a_{132}-256 a_{130} a_{133}+1024 a_{134}\right) \leq 0
\end{aligned}
$$

and so, $L_{13}^{\prime \prime \prime}(t)>0$ and $L_{13}^{(4)}(t) \leq 0$.

In conclusion, $L_{13}$ satisfies all the conditions of Theorem 2.2 and therefore, the solution of (2.17) satisfies the inequality $\left|h_{13}(t)\right| \leq 4$. Using the Convolution Theorem, the solution of (2.15) is found as

$$
f_{13}(t)=h_{13}(t)-\gamma_{13} \int_{0}^{t} h_{13}(t-\tau) e^{-\gamma_{13} \tau} d \tau
$$

and hence

$$
\left|f_{13}(t)\right| \leq 4+4 \gamma_{13} \int_{0}^{t} e^{-\gamma_{13} \tau} d \tau=4-4\left(e^{-\gamma_{13} t}-1\right) \leq 8 .
$$

At least one function $K_{13}$ satisfying conditions (1)-(7) of Theorem 2.3 can be obtained using the following method: 
Example 2.3. If there exist the numbers $a_{130}, a_{131}, a_{132}, a_{133}, a_{134}>0$ satisfying conditions (2)-(7) of Theorem 2.3, then there exists at least one function $K_{13}$ of the form

$$
K_{13}(t)=\sum_{m=0}^{4} c_{m} t^{4-m}+c_{5} e^{-t}
$$

such that

$$
K_{13}(0)=a_{130}, K_{13}^{\prime}(0)=a_{131}, K_{13}^{\prime \prime}(0)=a_{132}, K_{13}^{\prime \prime \prime}(0)=a_{133}, K_{13}^{(4)}(0)=a_{134}
$$

and $K_{13}$ also satisfies condition (1) of Theorem 2.3.

To see the validity of this assertion let us first choose the numbers $a_{130}$ and $a_{131}$ satisfying conditions (2) and (3) of Theorem 2.3 and define the polynomial $P_{13 i}$ for all $i \in \mathbb{N}_{5}$ and the number $\gamma_{13}$, as follows:

$$
P_{13 i}(\gamma)=\sum_{k=0}^{i}(-\gamma)^{i-k} a_{13(k-1)}\left(a_{13(-1)} \equiv 1 \text {, by convention) and } \gamma_{13}=\frac{a_{130}}{4}\right. \text {. }
$$

Then, it can be clearly seen from the proof of Theorem 2.3 that the numbers $a_{120}$ and $a_{121}$ defined by the equalities

$$
a_{120}=P_{131}\left(\gamma_{13}\right) \text { and } a_{121}=P_{132}\left(\gamma_{13}\right)
$$

satisfy conditions (2) and (3) of Theorem 2.2. It is of course that the numbers $a_{122}, a_{123}$ can also be found by means of $a_{120}, a_{121}$ from Example 2.2.

Besides, since condition (4) of Theorem 2.3 is equivalent to

$$
106\left[P_{131}\left(\gamma_{13}\right)\right]^{3}-405 P_{131}\left(\gamma_{13}\right) P_{132}\left(\gamma_{13}\right)+729 P_{133}\left(\gamma_{13}\right) \leq 0,
$$

if $P_{133}\left(\gamma_{13}\right)$ is taken as

$$
P_{133}\left(\gamma_{13}\right)=-\gamma_{13} P_{132}\left(\gamma_{13}\right)+a_{132}=a_{122},
$$

then the number $a_{132}$ is obtained as

$$
a_{132}=a_{122}+\gamma_{13} P_{132}\left(\gamma_{13}\right)=a_{122}+\gamma_{13} a_{121}>0 .
$$

Since condition (5) of Theorem 2.3 is equivalent to

$-2\left[P_{131}\left(\gamma_{13}\right)\right]^{4}+9\left[P_{131}\left(\gamma_{13}\right)\right]^{2} P_{132}\left(\gamma_{13}\right)-27 P_{131}\left(\gamma_{13}\right) P_{133}\left(\gamma_{13}\right)+81 P_{134}\left(\gamma_{13}\right) \leq 0$,

if $P_{134}\left(\gamma_{13}\right)$ is taken as

$$
P_{134}\left(\gamma_{13}\right)=-\gamma_{13} P_{133}\left(\gamma_{13}\right)+a_{133}=a_{123},
$$

then the number $a_{133}$ is found as

$$
a_{133}=a_{123}+\gamma_{13} P_{133}\left(\gamma_{13}\right)=a_{123}+\gamma_{13} a_{122}>0 .
$$

Condition (6) of Theorem 2.3 is equivalent to $P_{133}\left(\gamma_{13}\right)>0$ and since $P_{133}\left(\gamma_{13}\right)$ is equal to $a_{122}$ which is positive, the numbers $a_{130}, a_{131}$ and $a_{132}$, found as above, satisfy condition (6) of Theorem 2.3, as well.

Finally, since condition ( 7 ) of Theorem 2.3 is equivalent to

$$
P_{135}\left(\gamma_{13}\right)=-\gamma_{13} P_{134}\left(\gamma_{13}\right)+a_{134} \leq 0
$$

the number $a_{134}>0$ can be chosen as

$$
0<a_{134} \leq \gamma_{13} P_{134}\left(\gamma_{13}\right)=\gamma_{13} a_{123} .
$$


The numbers $a_{130}, a_{131}, a_{132}, a_{133}, a_{134}>0$ obtained by using the above method satisfy conditions (2)-(7) of Theorem 2.3. The solution of the system of linear equations (2.23) which is equivalent to

$c_{4}+c_{5}=a_{130}, c_{3}-c_{5}=a_{131}, 2 c_{2}+c_{5}=a_{132}, 6 c_{1}-c_{5}=a_{133}, 24 c_{0}+c_{5}=a_{134}$

is obtained as

$\left(c_{0}, c_{1}, c_{2}, c_{3}, c_{4}, c_{5}\right)=\left(\frac{a_{134}-c_{5}}{24}, \frac{a_{133}+c_{5}}{6}, \frac{a_{132}-c_{5}}{2}, a_{131}+c_{5}, a_{130}-c_{5}, c_{5}\right)$,

where $c_{5}$ is an arbitrary constant such that

$$
0 \leq c_{5}<\min \left\{a_{130}, a_{132}, a_{134}\right\} .
$$

So, every function $K_{13}$ of the form (2.22) also satisfies condition (1) of Theorem 2.3. For example, if the numbers $a_{130}$ and $a_{131}$ are chosen as $a_{130}=\frac{15}{2}$ and $a_{131}=21$, then

From Example 2.2,

$$
\gamma_{13}=\frac{15}{8}, a_{120}=P_{131}\left(\gamma_{13}\right)=\frac{45}{8}, a_{121}=P_{132}\left(\gamma_{13}\right)=\frac{669}{64} .
$$

$$
\gamma_{12}=\frac{15}{8}, a_{110}=P_{121}\left(\gamma_{12}\right)=\frac{15}{4}, a_{111}=P_{122}\left(\gamma_{12}\right)=\frac{219}{64} .
$$

From Example 2.1, $\gamma_{11}=\frac{5}{4}$ and the number $a_{112}$ satisfying the inequality

$$
0<a_{112} \leq \gamma_{11} P_{112}\left(\gamma_{11}\right)=\frac{95}{256}
$$

can be taken as $a_{112}=\frac{3}{10}$. Thus, from Example 2.2,

$$
a_{122}=a_{112}+\gamma_{12} a_{111}=\frac{32853}{10} ;
$$

the number $a_{123}$ satisfying the inequality

$$
0<a_{123} \leq \gamma_{12} a_{112}=\frac{9}{16}
$$

can be taken as $a_{123}=\frac{1}{2}$. Hence,

$$
\begin{aligned}
& a_{132}=a_{122}+\gamma_{13} a_{121}=\frac{8460543}{2560}, \\
& a_{133}=a_{123}+\gamma_{13} a_{122}=\frac{98567}{16} .
\end{aligned}
$$

Furthermore, the number $a_{134}$ satisfying the inequality

$$
0<a_{134} \leq \gamma_{13} a_{123}=\frac{15}{16}
$$

can be chosen as $a_{134}=\frac{9}{10}$. The number $c_{5}$ satisfying the inequality

$$
0 \leq c_{5}<\min \left\{a_{130}, a_{132}, a_{134}\right\}=\min \left\{\frac{15}{2}, \frac{8460543}{2560}, \frac{9}{10}\right\}
$$

can be chosen as $c_{5}=\frac{4}{5}$. Thus, we have

$$
\left(c_{0}, c_{1}, c_{2}, c_{3}, c_{4}, c_{5}\right)=\left(\frac{1}{240}, \frac{492899}{480}, \frac{1691699}{1024}, \frac{109}{5}, \frac{67}{10}, \frac{4}{5}\right)
$$

and

$$
K_{13}(t)=\frac{1}{240} t^{4}+\frac{492899}{480} t^{3}+\frac{1691699}{1024} t^{2}+\frac{109}{5} t+\frac{67}{10}+\frac{4}{5} e^{-t}
$$


Theorem 2.4. Let us consider the equation

$$
f_{14}(t)=1-\int_{0}^{t} K_{14}(t-\tau) f_{14}(\tau) d \tau=1-K_{14} * f_{14} .
$$

Suppose that the conditions

(1) $K_{14}(t)>0, K_{14}^{\prime}(t)>0, K_{14}^{\prime \prime}(t)>0, K_{14}^{\prime \prime \prime}(t)>0, K_{14}^{(4)}(t)>0, K_{14}^{(5)}(t)>0$ and $K_{14}^{(6)}(t) \leq 0$ for $0 \leq t<\infty$,

(2) $a_{140}^{2}<\frac{48}{19} a_{141}$

(3) $\frac{5}{2} a_{141} \leq a_{140}^{2}$,

(4) $718 a_{140}^{3}-2475 a_{140} a_{141}+3375 a_{142} \leq 0$,

(5) $\quad-7 a_{140}^{4}+30 a_{140}^{2} a_{141}-75 a_{140} a_{142}+125 a_{143} \leq 0$,

(6) $11 a_{140}^{3}-50 a_{140} a_{141}+125 a_{142}>0$,

(7) $19 a_{140}^{5}-100 a_{140}^{3} a_{141}+375 a_{140}^{2} a_{142}-1250 a_{140} a_{143}+3125 a_{144} \leq 0$,

(8) $4 a_{140}^{3}-25 a_{140} a_{141}+125 a_{142}>0$,

(9) $-4 a_{140}^{4}+25 a_{140}^{2} a_{141}-125 a_{140} a_{142}+625 a_{143}>0$,

(10) $-4 a_{140}^{6}+25 a_{140}^{4} a_{141}-125 a_{140}^{3} a_{142}+625 a_{140}^{2} a_{143}-3125 a_{140} a_{144}$

$+15625 a_{145} \leq 0$,

hold. Then the solution of (2.24) satisfies the inequality $\left|f_{14}(t)\right| \leq 16$ for $0 \leq t<\infty$, where $a_{140}=K_{14}(0), a_{141}=K_{14}^{\prime}(0), a_{142}=K_{14}^{\prime \prime}(0), a_{143}=K_{14}^{\prime \prime \prime}(0), a_{144}=$ $K_{14}^{(4)}(0), a_{145}=K_{14}^{(5)}(0)$.

Proof. If the function $g$ is taken as $g(t)=e^{-\gamma t}(\gamma \in \mathbb{R})$ in Theorem A, then the equation which is equivalent to (2.24) is found as

$$
f_{14}(t)=e^{-\gamma t}-L_{14} * f_{14},
$$

where

$$
L_{14}(t)=\left(a_{140}-\gamma\right) e^{-\gamma t}+K_{14}^{\prime} * e^{-\gamma t} .
$$

By differentiating (2.26), it is obtained by $L_{14}^{\prime}(t), L_{14}^{\prime \prime}(t), L_{14}^{\prime \prime \prime}(t), L_{14}^{(4)}(t)$ and $L_{14}^{(5)}(t)$ that

$$
\begin{aligned}
L_{14}^{\prime}(t) & =\left(\gamma^{2}-a_{140} \gamma+a_{141}\right) e^{-\gamma t}+K_{14}^{\prime \prime} * e^{-\gamma t} \\
L_{14}^{\prime \prime}(t) & =\left(-\gamma^{3}+a_{140} \gamma^{2}-a_{141} \gamma+a_{142}\right) e^{-\gamma t}+K_{14}^{\prime \prime \prime} * e^{-\gamma t} \\
L_{14}^{\prime \prime \prime}(t) & =\left(\gamma^{4}-a_{140} \gamma^{3}+a_{141} \gamma^{2}-a_{142} \gamma+a_{143}\right) e^{-\gamma t}+K_{14}^{(4)} * e^{-\gamma t} \\
L_{14}^{(4)}(t) & =\left(-\gamma^{5}+a_{140} \gamma^{4}-a_{141} \gamma^{3}+a_{142} \gamma^{2}-a_{143} \gamma+a_{144}\right) e^{-\gamma t}+K_{14}^{(5)} * e^{-\gamma t}
\end{aligned}
$$

and

$L_{14}^{(5)}(t)=\left(\gamma^{6}-a_{140} \gamma^{5}+a_{141} \gamma^{4}-a_{142} \gamma^{3}+a_{143} \gamma^{2}-a_{144} \gamma+a_{145}\right) e^{-\gamma t}+K_{14}^{(6)} * e^{-\gamma t}$.

Now, one can see that the kernel $L_{14}$ of the equation

$$
h_{14}(t)=1-L_{14} * h_{14}
$$

satisfies the conditions of Theorem 2.3. 
The corresponding inequalities to conditions (2) and (3) of Theorem 2.3 are

$$
\left[L_{14}(0)\right]^{2}<\frac{19}{7} L_{14}^{\prime}(0)
$$

and

$$
\frac{8}{3} L_{14}^{\prime}(0) \leq\left[L_{14}(0)\right]^{2}
$$

respectively. Inequalities (2.28) and (2.29) are equivalent to

$$
\left(a_{140}-\gamma\right)^{2}<\frac{19}{7}\left(\gamma^{2}-a_{140} \gamma+a_{141}\right)
$$

and

$$
\frac{8}{3}\left(\gamma^{2}-a_{140} \gamma+a_{141}\right) \leq\left(a_{140}-\gamma\right)^{2},
$$

respectively. Inequality (2.30) is equivalent to

$$
q_{1}(\gamma)=12 \gamma^{2}-5 a_{140} \gamma+19 a_{141}-7 a_{140}^{2}>0 .
$$

The discriminant of $q_{1}(\gamma)=0$ is $19\left(19 a_{140}^{2}-48 a_{141}\right)$ which is negative by condition (2) of Theorem 2.4. Hence, $q_{1}(\gamma)>0$ for every $\gamma \in \mathbb{R}$.

Inequality (2.31) is equivalent to

$$
q_{2}(\gamma)=5 \gamma^{2}-2 a_{140} \gamma+8 a_{141}-3 a_{140}^{2} \leq 0 .
$$

The discriminant of $q_{2}(\gamma)=0$ is $32\left(2 a_{140}^{2}-5 a_{141}\right)$, which is nonnegative by condition (3) of Theorem 2.4. Hence, $q_{2}\left(\gamma_{14}\right) \leq 0$ whenever $\gamma$ is chosen as $\gamma=\gamma_{14}=\frac{a_{140}}{5}$.

The corresponding inequalities to conditions (4)-(7) of Theorem 2.3 are

$$
\begin{gathered}
161\left[L_{14}(0)\right]^{3}-576 L_{14}(0) L_{14}^{\prime}(0)+864 L_{14}^{\prime \prime}(0) \leq 0, \\
-11\left[L_{14}(0)\right]^{4}+48\left[L_{14}(0)\right]^{2} L_{14}^{\prime}(0)-128 L_{14}(0) L_{14}^{\prime \prime}(0)+256 L_{14}^{\prime \prime \prime}(0) \leq 0, \\
3\left[L_{14}(0)\right]^{3}-16 L_{14}(0) L_{14}^{\prime}(0)+64 L_{14}^{\prime \prime}(0)>0, \\
3\left[L_{14}(0)\right]^{5}-16\left[L_{14}(0)\right]^{3} L_{14}^{\prime}(0)+64\left[L_{14}(0)\right]^{2} L_{14}^{\prime \prime}(0) \\
-256 L_{14}(0) L_{14}^{\prime \prime \prime}(0)+1024 L_{14}^{(4)}(0) \leq 0,
\end{gathered}
$$

respectively. These inequalities are equivalent to

$$
\begin{aligned}
q_{3}(\gamma)= & 161\left(a_{140}-\gamma\right)^{3}-576\left(a_{140}-\gamma\right)\left(\gamma^{2}-a_{140} \gamma+a_{141}\right) \\
& +864\left(-\gamma^{3}+a_{140} \gamma^{2}-a_{141} \gamma+a_{142}\right) \leq 0 \\
q_{4}(\gamma)= & -11\left(a_{140}-\gamma\right)^{4}+48\left(a_{140}-\gamma\right)^{2}\left(\gamma^{2}-a_{140} \gamma+a_{141}\right) \\
& -128\left(a_{140}-\gamma\right)\left(-\gamma^{3}+a_{140} \gamma^{2}-a_{141} \gamma+a_{142}\right) \\
& +256\left(\gamma^{4}-a_{140} \gamma^{3}+a_{141} \gamma^{2}-a_{142} \gamma+a_{143}\right) \leq 0 \\
q_{5}(\gamma)= & 3\left(a_{140}-\gamma\right)^{3}-16\left(a_{140}-\gamma\right)\left(\gamma^{2}-a_{140} \gamma+a_{141}\right) \\
& +64\left(-\gamma^{3}+a_{140} \gamma^{2}-a_{141} \gamma+a_{142}\right)>0 \\
q_{6}(\gamma)= & 3\left(a_{140}-\gamma\right)^{5}-16\left(a_{140}-\gamma\right)^{3}\left(\gamma^{2}-a_{140} \gamma+a_{141}\right) \\
& +64\left(a_{140}-\gamma\right)^{2}\left(-\gamma^{3}+a_{140} \gamma^{2}-a_{141} \gamma+a_{142}\right) \\
& -256\left(a_{140}-\gamma\right)\left(\gamma^{4}-a_{140} \gamma^{3}+a_{141} \gamma^{2}-a_{142} \gamma+a_{143}\right) \\
& +1024\left(-\gamma^{5}+a_{140} \gamma^{4}-a_{141} \gamma^{3}+a_{142} \gamma^{2}\right. \\
& \left.-a_{143} \gamma+a_{144}\right) \leq 0,
\end{aligned}
$$


respectively. By conditions (4), (5), (6) and (7) of Theorem 2.4, respectively, it is obtained by $q_{3}\left(\gamma_{14}\right), q_{4}\left(\gamma_{14}\right), q_{5}\left(\gamma_{14}\right)$ and $q_{6}\left(\gamma_{14}\right)$ that

$$
\begin{aligned}
& q_{3}\left(\gamma_{14}\right)=\frac{32}{125}\left(718 a_{140}^{3}-2475 a_{140} a_{141}+3375 a_{142}\right) \leq 0, \\
& q_{4}\left(\gamma_{14}\right)=\frac{256}{125}\left(-7 a_{140}^{4}+30 a_{140}^{2} a_{141}-75 a_{140} a_{142}+125 a_{143}\right) \leq 0, \\
& q_{5}\left(\gamma_{14}\right)=\frac{64}{125}\left(11 a_{140}^{3}-50 a_{140} a_{141}+125 a_{142}\right)>0
\end{aligned}
$$

and

$$
q_{6}\left(\gamma_{14}\right)=\frac{1024}{3125}\left(19 a_{140}^{5}-100 a_{140}^{3} a_{141}+375 a_{140}^{2} a_{142}-1250 a_{140} a_{143}+3125 a_{144}\right) \leq 0 .
$$

The corresponding inequalities to condition (1) of Theorem 2.3 are $L_{14}(t), L_{14}^{\prime}(t)$, $L_{14}^{\prime \prime}(t), L_{14}^{\prime \prime \prime}(t), L_{14}^{(4)}(t)>0, L_{14}^{(5)}(t) \leq 0$.

Since $a_{140}-\gamma_{14}$ is positive, $L_{14}(t)>0$ and the discriminant of $\gamma^{2}-a_{140} \gamma+a_{141}=0$ is negative by condition (2) of Theorem 2.4, $\gamma_{14}^{2}-a_{140} \gamma_{14}+a_{141}>0$ and so, $L_{14}^{\prime}(t)>0$.

By condition (8) of Theorem 2.4,

$$
-\gamma_{14}^{3}+a_{140} \gamma_{14}^{2}-a_{141} \gamma_{14}+a_{142}=\frac{1}{125}\left(4 a_{140}^{3}-25 a_{140} a_{141}+125 a_{142}\right)>0 .
$$

Therefore, $L_{14}^{\prime \prime}(t)>0$.

By condition (9) of Theorem 2.4,

$$
\begin{aligned}
& \gamma_{14}^{4}-a_{140} \gamma_{14}^{3}+a_{141} \gamma_{14}^{2}-a_{142} \gamma_{14}+a_{143} \\
& =\frac{1}{625}\left(-4 a_{140}^{4}+25 a_{140}^{2} a_{141}-125 a_{140} a_{142}+625 a_{143}\right)>0 .
\end{aligned}
$$

Thus, $L_{14}^{\prime \prime \prime}(t)>0$.

From condition (10) of Theorem 2.4,

$$
\begin{gathered}
-\gamma_{14}^{5}+a_{140} \gamma_{14}^{4}-a_{141} \gamma_{14}^{3}+a_{142} \gamma_{14}^{2}-a_{143} \gamma_{14}+a_{144} \\
=\frac{1}{3125}\left(4 a_{140}^{5}-25 a_{140}^{3} a_{141}+125 a_{140}^{2} a_{142}-625 a_{140} a_{143}+3125 a_{144}\right)>0 \\
\gamma_{14}^{6}-a_{140} \gamma_{14}^{5}+a_{141} \gamma_{14}^{4}-a_{142} \gamma_{14}^{3}+a_{143} \gamma_{14}^{2}-a_{144} \gamma_{14}+a_{145} \\
=\frac{1}{15625}\left(-4 a_{140}^{6}+25 a_{140}^{4} a_{141}-125 a_{140}^{3} a_{142}\right. \\
\left.+625 a_{140}^{2} a_{143}-3125 a_{140} a_{144}+15625 a_{145}\right) \leq 0
\end{gathered}
$$

So, $L_{14}^{(4)}(t)>0, L_{14}^{(5)}(t) \leq 0$. Hence, the solution of (2.27) satisfies the inequality $\left|h_{14}(t)\right| \leq 8$ by Theorem 2.3. By Theorem B, the solution of (2.25) can be expressed as

and thus,

$$
f_{14}(t)=h_{14}(t)-\gamma_{14} \int_{0}^{t} h_{14}(t-\tau) e^{-\gamma_{14} \tau} d \tau
$$

$$
\left|f_{14}(t)\right| \leq 8+8 \gamma_{14} \int_{0}^{t} e^{-\gamma_{14} \tau} d \tau=8-8\left(e^{-\gamma_{14} t}-1\right) \leq 16
$$

which completes the proof of Theorem 2.4.

A function $K_{14}$ satisfying conditions (1)-(10) of Theorem 2.4 can be obtained by the following method: 
Example 2.4. If there exist the numbers $a_{140}, a_{141}, a_{142}, a_{143}, a_{144}, a_{145}>0$ satisfying conditions (2)-(10) of Theorem 2.4, then there exists at least one function $K_{14}$ which satisfies condition (1) of Theorem 2.4 in the form

$$
K_{14}(t)=\sum_{m=0}^{5} c_{m} t^{5-m}+c_{6} e^{-t}
$$

such that

$$
\begin{gathered}
K_{14}(0)=a_{140}, K_{14}^{\prime}(0)=a_{141}, K_{14}^{\prime \prime}(0)=a_{142}, \\
K_{14}^{\prime \prime \prime}(0)=a_{143}, K_{14}^{(4)}(0)=a_{144}, K_{14}^{(5)}(0)=a_{145} .
\end{gathered}
$$

First, let us show that there exist the numbers $a_{140}, a_{141}, a_{142}, a_{143}, a_{144}$, $a_{145}>0$ satisfying conditions (2)-(10) of Theorem 2.4. It is clear that the numbers $a_{140}$ and $a_{141}$ satisfying conditions (2) and (3) of Theorem 2.4 exist.

Now, we shall define the polynomial $P_{14 i}$ for all $i \in \mathbb{N}_{6}$ and the number $\gamma_{14}$ by

$$
P_{14 i}(\gamma)=\sum_{k=0}^{i}(-\gamma)^{i-k} a_{14(k-1)}\left(a_{14(-1)} \equiv 1 \text {, by convention) and } \gamma_{14}=\frac{a_{140}}{5}\right. \text {. }
$$

Then, it can be clearly observed from the proof of Theorem 2.4 that the numbers $a_{130}$ and $a_{131}$ defined by

$$
a_{130}=P_{141}\left(\gamma_{14}\right) \text { and } a_{131}=P_{142}\left(\gamma_{14}\right)
$$

satisfy conditions (2) and (3) of Theorem 2.3. Besides, the numbers $a_{132}, a_{133}, a_{134}$ can also be found by means of $a_{130}$ and $a_{131}$ as those were found in Example 2.3. Since condition (4) of Theorem 2.4 is equivalent to

$$
161\left[P_{141}\left(\gamma_{14}\right)\right]^{3}-576 P_{141}\left(\gamma_{14}\right) P_{142}\left(\gamma_{14}\right)+864 P_{143}\left(\gamma_{14}\right) \leq 0,
$$

if $P_{143}\left(\gamma_{14}\right)$ is taken as

$$
P_{143}\left(\gamma_{14}\right)=-\gamma_{14} P_{142}\left(\gamma_{14}\right)+a_{142}=a_{132},
$$

then the number $a_{142}$ is obtained as

$$
a_{142}=a_{132}+\gamma_{14} P_{142}\left(\gamma_{14}\right)=a_{132}+\gamma_{14} a_{131}>0 .
$$

Since condition (5) of Theorem 2.4 is equivalent to

$-11\left[P_{141}\left(\gamma_{14}\right)\right]^{4}+48\left[P_{141}\left(\gamma_{14}\right)\right]^{2} P_{142}\left(\gamma_{14}\right)-128 P_{141}\left(\gamma_{14}\right) P_{143}\left(\gamma_{14}\right)+256 P_{144}\left(\gamma_{14}\right) \leq 0$, if $P_{144}\left(\gamma_{14}\right)$ is taken as

$$
P_{144}\left(\gamma_{14}\right)=-\gamma_{14} P_{143}\left(\gamma_{14}\right)+a_{143}=a_{133},
$$

then the number $a_{143}$ is found as

$$
a_{143}=a_{133}+\gamma_{14} P_{143}\left(\gamma_{14}\right)=a_{133}+\gamma_{14} a_{132}>0 .
$$

Because condition (6) of Theorem 2.4 is equivalent to

$3\left[P_{141}\left(\gamma_{14}\right)\right]^{3}-16 P_{141}\left(\gamma_{14}\right) P_{142}\left(\gamma_{14}\right)+64 P_{143}\left(\gamma_{14}\right)=3 a_{130}^{3}-16 a_{130} a_{131}+64 a_{132}>0$ and $3 a_{130}^{3}-16 a_{130} a_{131}+64 a_{132}$ is positive from Example 2.3, the numbers $a_{140}, a_{141}$, $a_{142}$, found as above, satisfy condition (6) of Theorem 2.4, as well. Since condition (7) of Theorem 2.4 is equivalent to

$$
\begin{gathered}
3\left[P_{141}\left(\gamma_{14}\right)\right]^{5}-16\left[P_{141}\left(\gamma_{14}\right)\right]^{3} P_{142}\left(\gamma_{14}\right)+64\left[P_{141}\left(\gamma_{14}\right)\right]^{2} P_{143}\left(\gamma_{14}\right) \\
-256 P_{141}\left(\gamma_{14}\right) P_{144}\left(\gamma_{14}\right)+1024 P_{145}\left(\gamma_{14}\right) \leq 0
\end{gathered}
$$


if $P_{145}\left(\gamma_{14}\right)$ is taken as

$$
P_{145}\left(\gamma_{14}\right)=-\gamma_{14} P_{144}\left(\gamma_{14}\right)+a_{144}=a_{134},
$$

then the number $a_{144}$ is found as

$$
a_{144}=a_{134}+\gamma_{14} P_{144}\left(\gamma_{14}\right)=a_{134}+\gamma_{14} a_{133}>0 .
$$

Since condition (8) of Theorem 2.4 is equivalent to $P_{143}\left(\gamma_{14}\right)>0$ and $P_{143}\left(\gamma_{14}\right)$ is equal to $a_{132}$, the numbers $a_{140}, a_{141}, a_{142}$, obtained as above, satisfy condition (8) of Theorem 2.4, as well. Because condition (9) of Theorem 2.4 is equivalent to $P_{144}\left(\gamma_{14}\right)>0$ and $P_{144}\left(\gamma_{14}\right)$ is equal to $a_{133}$, the numbers $a_{140}, a_{141}, a_{142}, a_{143}$, found as above, satisfy condition (9) of Theorem 2.4, as well.

Finally, since condition (10) of Theorem 2.4 is equivalent to

$$
P_{146}\left(\gamma_{14}\right)=-\gamma_{14} P_{145}\left(\gamma_{14}\right)+a_{145} \leq 0,
$$

the number $a_{145}>0$ which is chosen as

$$
0<a_{145} \leq \gamma_{14} P_{145}\left(\gamma_{14}\right)=\gamma_{14} a_{134}
$$

and the numbers $a_{140}, a_{141}, a_{142}, a_{143}, a_{144}$ satisfy condition (10) of Theorem 2.4.

Clearly, the obtained numbers $a_{140}, a_{141}, a_{142}, a_{143}, a_{144}$ and $a_{145}$ by presented method satisfy conditions (2)-(10) of Theorem 2.4.

The solution of the system of linear equations (2.33) which is equivalent to

$$
\begin{gathered}
c_{5}+c_{6}=a_{140}, c_{4}-c_{6}=a_{141}, 2 c_{3}+c_{6}=a_{142}, \\
6 c_{2}-c_{6}=a_{143}, 24 c_{1}+c_{6}=a_{144}, 120 c_{0}-c_{6}=a_{145}
\end{gathered}
$$

is obtained as

$$
\begin{aligned}
& \left(c_{0}, c_{1}, c_{2}, c_{3}, c_{4}, c_{5}, c_{6}\right) \\
& \quad=\left(\frac{a_{145}+c_{6}}{120}, \frac{a_{144}-c_{6}}{24}, \frac{a_{143}+c_{6}}{6}, \frac{a_{142}-c_{6}}{2}, a_{141}+c_{6}, a_{140}-c_{6}, c_{6}\right),
\end{aligned}
$$

where $c_{6}$ is an arbitrary constant satisfying the inequality

$$
\max \left\{-a_{141},-a_{143},-a_{145}\right\}<c_{6} \leq 0 .
$$

Thus, every function $K_{14}$ of the form (2.32) also satisfies condition (1) of Theorem 2.4. For example, if the numbers $a_{140}$ and $a_{141}$ are taken as $a_{140}=\frac{173}{25}$ and $a_{141}=19$, then $\gamma_{14}, a_{130}, a_{131}$ are obtained as

$$
\gamma_{14}=\frac{173}{125}, a_{130}=P_{141}\left(\gamma_{14}\right)=\frac{692}{125}, a_{131}=P_{142}\left(\gamma_{14}\right)=\frac{177159}{15625} .
$$

From Examples 2.3 and 2.2, respectively, we derive that

and

$$
\gamma_{13}=\frac{173}{125}, a_{120}=P_{131}\left(\gamma_{13}\right)=\frac{519}{125}, a_{121}=P_{132}\left(\gamma_{13}\right)=\frac{87372}{15625}
$$

$$
\gamma_{12}=\frac{173}{125}, a_{110}=P_{121}\left(\gamma_{12}\right)=\frac{346}{125}, a_{111}=P_{122}\left(\gamma_{12}\right)=\frac{27514}{15625} .
$$

From Example 2.1, $\gamma_{11}=\frac{346}{375}$, and the positive number $a_{112}$ satisfying the inequality

$$
0<a_{112} \leq \gamma_{11} P_{112}\left(\gamma_{11}\right)=\frac{2835124}{52734375}
$$

can be taken as $a_{112}=\frac{1}{25}$. Hence, one can derive from Example 2.2 that

$$
a_{122}=a_{112}+\gamma_{12} a_{111}=\frac{4338047}{1953125}
$$


and the number $a_{123}$ satisfying the inequality

$$
0<a_{123} \leq \gamma_{12} a_{112}=\frac{173}{3125}
$$

can be taken as $a_{123}=\frac{1}{25}$. From Example 2.3, we have that

$$
\begin{aligned}
& a_{132}=a_{122}+\gamma_{13} a_{121}=\frac{19953403}{1953125} \\
& a_{133}=a_{123}+\gamma_{13} a_{122}=\frac{846747756}{244140625} .
\end{aligned}
$$

Furthermore, the number $a_{134}$ satisfying the inequality

$$
0<a_{134} \leq \gamma_{13} a_{123}=\frac{173}{3125}
$$

can be chosen as $a_{134}=\frac{1}{25}$. So,

$$
\begin{aligned}
& a_{142}=a_{132}+\gamma_{14} a_{131}=\frac{10120382}{390625}, \\
& a_{143}=a_{133}+\gamma_{14} a_{132}=\frac{171947459}{9765625}, \\
& a_{144}=a_{134}+\gamma_{14} a_{133}=\frac{147708064913}{30517578125}
\end{aligned}
$$

and the number $a_{145}$ satisfying the inequality

$$
0<a_{145} \leq \gamma_{14} a_{134}=\frac{173}{3125}
$$

can be chosen as $a_{145}=\frac{1}{25}$. The number $c_{6}$ satisfying the inequality

$$
\max \left\{-a_{141},-a_{143},-a_{145}\right\}=\max \left\{-19,-\frac{171947459}{9765625},-\frac{1}{25}\right\}<c_{6} \leq 0
$$

can be chosen as $c_{6}=-\frac{1}{125}$. Thus, we have

$$
\begin{aligned}
& \left(c_{0}, c_{1}, c_{2}, c_{3}, c_{4}, c_{5}, c_{6}\right) \\
& \quad=\left(\frac{1}{3750}, \frac{73976102769}{366210937500}, \frac{28644889}{9765625}, \frac{10123507}{781250}, \frac{2374}{125}, \frac{866}{125},-\frac{1}{125}\right)
\end{aligned}
$$

and

$$
\begin{aligned}
K_{14}(t)= & \frac{1}{3750} t^{5}+\frac{73976102769}{366210937500} t^{4}+\frac{28644889}{9765625} t^{3} \\
& +\frac{10123507}{781250} t^{2}+\frac{2374}{125} t+\frac{866}{125}-\frac{1}{125} e^{-t}
\end{aligned}
$$

By continuing this process for $n \in \mathbb{N}$, we have Theorem 2.n and Example 2.n which may be stated, as follows:

Theorem 2.n. Let us consider the equation of the form

$$
f_{1 n}(t)=1-\int_{0}^{t} K_{1 n}(t-\tau) f_{1 n}(\tau) d \tau=1-K_{1 n} * f_{1 n} .
$$


Suppose that the conditions

$$
\begin{aligned}
& \text { (1) } K_{1 n}(t)>0, K_{1 n}^{\prime}(t)>0, \ldots, K_{1 n}^{(n+1)}(t)>0 \text { and } K_{1 n}^{(n+2)}(t) \leq 0, \\
& \\
& \quad \text { for } 0 \leq t<\infty \\
& \text { (2) } a_{1 n 0}^{2}<\frac{2(5 n+4)}{5 n-1} a_{1 n 1} \\
& \text { (3) } \frac{2(n+1)}{n} a_{1 n 1} \leq a_{1 n 0}^{2}
\end{aligned}
$$

hold. Furthermore, we assume that conditions $(4)-\left(4+t_{1 n}\right)$ of Theorem 2.n are the inequalities obtained by taking

$$
P_{1 n 1}\left(\gamma_{1 n}\right), P_{1 n 2}\left(\gamma_{1 n}\right), \ldots, P_{1 n(n+1)}\left(\gamma_{1 n}\right),
$$

respectively instead of the constants $a_{1(n-1) 0}, a_{1(n-1) 1}, \ldots, a_{1(n-1) n}$ in conditions $(4)-\left(4+t_{1 n}\right)$ of Theorem 2. $(n-1)$ for $n \geq 2$. Let conditions $\left(5+t_{1 n}\right)-\left(2+n+t_{1 n}\right)$ of Theorem $2 . n$ be

$$
P_{1 n 3}\left(\gamma_{1 n}\right), P_{1 n 4}\left(\gamma_{1 n}\right), \ldots, P_{1 n n}\left(\gamma_{1 n}\right)>0 \text { for } n \geq 3
$$

respectively. Additionally, let condition $\left(3+n+t_{1 n}\right)$ of Theorem $2 . n$ be

$$
P_{1 n(n+2)}\left(\gamma_{1 n}\right) \leq 0 \text { for } n \geq 1,
$$

where

$$
\begin{gathered}
a_{1 n 0}=K_{1 n}(0), a_{1 n 1}=K_{1 n}^{\prime}(0), a_{1 n 2}=K_{1 n}^{\prime \prime}(0), \ldots, a_{1 n(n+1)}=K_{1 n}^{(n+1)}(0), \\
t_{1 n}=\frac{(n-2)(n-1)}{2} \\
P_{1 n i}(\gamma)=\sum_{k=0}^{i}(-\gamma)^{i-k} a_{1 n(k-1)},\left(a_{1 n(-1)} \equiv 1, \quad \text { by convention }\right) \text { for all } i \in \mathbb{N}_{n+2}
\end{gathered}
$$

and

$$
\gamma_{11}=\frac{a_{110}}{3} \text { for } n=1 \text { and } \gamma_{1 n}=\frac{a_{1 n 0}}{n+1} \text { for } n \geq 2 .
$$

Then, the solution of (2.34) satisfies the inequality $\left|f_{1 n}(t)\right| \leq 2^{n}$ for $0 \leq t<\infty$.

Proof. We prove the theorem by using the induction method.

The validity of Theorem 2.1 is known by 3 .

Let us suppose the truth of Theorem $2 . m$ for $m \in \mathbb{N}$. So, the solution of the equation

$$
f_{1 m}(t)=1-K_{1 m} * f_{1 m}
$$

satisfies the inequality

$$
\left|f_{1 m}(t)\right| \leq 2^{m} .
$$

Now, we should prove that Theorem 2. $(\mathrm{m}+1)$ is true. Namely, let us show that the solution of the equation

$$
f_{1(m+1)}(t)=1-K_{1(m+1)} * f_{1(m+1)}
$$

satisfies the condition

$$
\left|f_{1(m+1)}(t)\right| \leq 2^{m+1}
$$


If the function $g$ is taken as $g(t)=e^{-\gamma t}(\gamma \in \mathbb{R})$ in Theorem $\mathrm{A}$, then one can see that

$$
f_{1(m+1)}(t)=e^{-\gamma t}-L_{1(m+1)} * f_{1(m+1)}
$$

is equivalent to (2.36), where

$$
L_{1(m+1)}(t)=\left(a_{1(m+1) 0}-\gamma\right) e^{-\gamma t}+K_{1(m+1)}^{\prime} * e^{-\gamma t} .
$$

By differentiating (2.39) $, L_{1(m+1)}^{\prime}(t), L_{1(m+1)}^{\prime \prime}(t), \ldots, L_{1(m+1)}^{(m+2)}(t)$ are found, as follows:

$$
\begin{aligned}
L_{1(m+1)}^{\prime}(t)= & \left(\gamma^{2}-a_{1(m+1) 0} \gamma+a_{1(m+1) 1}\right) e^{-\gamma t}+K_{1(m+1)}^{\prime \prime} * e^{-\gamma t}, \\
L_{1(m+1)}^{\prime \prime}(t)= & \left(-\gamma^{3}+a_{1(m+1) 0} \gamma^{2}-a_{1(m+1) 1} \gamma+a_{1(m+1) 2}\right) e^{-\gamma t}+K_{1(m+1)}^{\prime \prime \prime} * e^{-\gamma t}, \\
\vdots & \\
L_{1(m+1)}^{(m)}(t)= & \left((-1)^{m+1} \gamma^{m+1}+(-1)^{m} a_{1(m+1) 0} \gamma^{m}+(-1)^{m-1} a_{1(m+1) 1} \gamma^{m-1}\right. \\
& \left.+\cdots-a_{1(m+1)(m-1)} \gamma+a_{1(m+1) m}\right) e^{-\gamma t}+K_{1(m+1)}^{(m+1)} * e^{-\gamma t}, \\
L_{1(m+1)}^{(m+1)}(t)= & \left((-1)^{m+2} \gamma^{m+2}+(-1)^{m+1} a_{1(m+1) 0} \gamma^{m+1}+(-1)^{m} a_{1(m+1) 1} \gamma^{m}\right. \\
& \left.+\cdots-a_{1(m+1) m} \gamma+a_{1(m+1)(m+1)}\right) e^{-\gamma t}+K_{1(m+1)}^{(m+2)} * e^{-\gamma t}, \\
L_{1(m+1)}^{(m+2)}(t)= & \left((-1)^{m+3} \gamma^{m+3}+(-1)^{m+2} a_{1(m+1) 0} \gamma^{m+2}+(-1)^{m+1} a_{1(m+1) 1} \gamma^{m+1}\right. \\
& \left.+\cdots-a_{1(m+1)(m+1)} \gamma+a_{1(m+1)(m+2)}\right) e^{-\gamma t}+K_{1(m+1)}^{(m+3)} * e^{-\gamma t} .
\end{aligned}
$$

We claim that the kernel of the equation

$$
h_{1(m+1)}(t)=1-L_{1(m+1)} * h_{1(m+1)}
$$

satisfies the conditions of Theorem $2 . \mathrm{m}$. That is, $L_{1(m+1)}$ satisfies all the conditions of Theorem 2.m under the assumptions of Theorem 2. $(\mathrm{m}+1)$.

The corresponding inequalities to conditions (2) and (3) of Theorem 2.m are

$$
\left[L_{1(m+1)}(0)\right]^{2}<\frac{2(5 m+4)}{5 m-1} L_{1(m+1)}^{\prime}(0)
$$

and

$$
\frac{2(m+1)}{m} L_{1(m+1)}^{\prime}(0) \leq\left[L_{1(m+1)}(0)\right]^{2}
$$

respectively. Inequalities (2.41) and (2.42) are respectively equivalent to

$$
\left(a_{1(m+1) 0}-\gamma\right)^{2}<\frac{2(5 m+4)}{5 m-1}\left(\gamma^{2}-a_{1(m+1) 0} \gamma+a_{1(m+1) 1}\right)
$$

and

$$
\frac{2(m+1)}{m}\left(\gamma^{2}-a_{1(m+1) 0} \gamma+a_{1(m+1) 1}\right) \leq\left(a_{1(m+1) 0}-\gamma\right)^{2} .
$$

Inequality (2.43) is equivalent to

$q_{1}(\gamma)=(5 m+9) \gamma^{2}-10 a_{1(m+1) 0} \gamma+2(5 m+4) a_{1(m+1) 1}-(5 m-1) a_{1(m+1) 0}^{2}>0$.

The discriminant of $q_{1}(\gamma)=0$ is

$$
4(5 m+4)\left[(5 m+4) a_{1(m+1) 0}^{2}-2(5 m+9) a_{1(m+1) 1}\right]
$$


which is negative by condition (2) of Theorem 2. $(\mathrm{m}+1)$, and thus, $q_{1}(\gamma)>0$ for every $\gamma \in \mathbb{R}$. Inequality (2.44) is equivalent to

$$
q_{2}(\gamma)=(m+2) \gamma^{2}-2 a_{1(m+1) 0} \gamma-m a_{1(m+1) 0}^{2}+2(m+1) a_{1(m+1) 1} \leq 0 .
$$

The discriminant of $q_{2}(\gamma)=0$ is

$$
4(m+1)\left[(m+1) a_{1(m+1) 0}^{2}-2(m+2) a_{1(m+1) 1}\right],
$$

which is nonnegative by condition (3) of Theorem 2.(m+1), and so $q_{2}\left(\gamma_{1(m+1)}\right) \leq 0$ whenever $\gamma$ is chosen as $\gamma=\gamma_{1(m+1)}=\frac{a_{1(m+1) 0}}{m+2}$. The corresponding inequalities to conditions $(4)-\left(3+m+t_{1 m}\right)$ of Theorem $2 . m$ are the inequalities obtained by taking

$$
P_{1(m+1) 1}\left(\gamma_{1(m+1)}\right), P_{1(m+1) 2}\left(\gamma_{1(m+1)}\right), \ldots, P_{1(m+1)(m+2)}\left(\gamma_{1(m+1)}\right),
$$

respectively, instead of the constants $a_{1 m 0}, a_{1 m 1}, \ldots, a_{1 m(m+1)}$ in conditions (4) $\left(3+m+t_{1 m}\right)$ of Theorem 2.m. These inequalities are conditions $(4)-\left(4+t_{1(m+1)}\right)$ of Theorem 2. $(\mathrm{m}+1)$ for $m \geq 1$, respectively. Hence, $L_{1(m+1)}$ satisfies conditions (4) $-\left(4+t_{1 m}\right)$ of Theorem 2.m. Furthermore, the corresponding inequalities to condition (1) of Theorem 2.m are

$$
L_{1(m+1)}(t), L_{1(m+1)}^{\prime}(t), \ldots, L_{1(m+1)}^{(m+1)}(t)>0 \text { and } L_{1(m+1)}^{(m+2)}(t) \leq 0 .
$$

Since $P_{1(m+1) 1}\left(\gamma_{1(m+1)}\right)$ is positive, $L_{1(m+1)}(t)>0$ and the discriminant of

$$
P_{1(m+1) 2}(\gamma)=0
$$

is negative by condition (2) of Theorem 2.(m+1), $P_{1(m+1) 2}\left(\gamma_{1(m+1)}\right)>0$ and so, $L_{1(m+1)}^{\prime}(t)>0$. Additionally, conditions $\left(5+t_{1(m+1)}\right)-\left(3+m+t_{1(m+1)}\right)$ of Theorem 2. $(\mathrm{m}+1)$ are

$$
P_{1(m+1) 3}\left(\gamma_{1(m+1)}\right), P_{1(m+1) 4}\left(\gamma_{1(m+1)}\right), \ldots, P_{1(m+1)(m+1)}\left(\gamma_{1(m+1)}\right)>0 \text { for } m \geq 2,
$$

respectively. Therefore,

$$
L_{1(m+1)}^{\prime \prime}(t), L_{1(m+1)}^{\prime \prime \prime}(t), \ldots, L_{1(m+1)}^{(m)}(t)>0 \text { for } m \geq 2 .
$$

Condition $\left(4+m+t_{1(m+1)}\right)$ of Theorem 2. $(\mathrm{m}+1)$ is

$$
\begin{aligned}
P_{1(m+1)(m+3)}\left(\gamma_{1(m+1)}\right)= & -\gamma_{1(m+1)} P_{1(m+1)(m+2)}\left(\gamma_{1(m+1)}\right) \\
& +a_{1(m+1)(m+2)} \leq 0 \text { for } m \geq 1 .
\end{aligned}
$$

Hence,

$$
P_{1(m+1)(m+2)}\left(\gamma_{1(m+1)}\right)>0
$$

and thus,

$$
L_{1(m+1)}^{(m+1)}(t)>0, L_{1(m+1)}^{(m+2)}(t) \leq 0 \text { for } m \geq 1 .
$$

Since $L_{1(m+1)}$ satisfies all the conditions of Theorem 2.m, the solution of the equation (2.40) satisfies (2.35). Namely, $\left|h_{1(m+1)}(t)\right| \leq 2^{m}$.

By Theorem B, the solution of (2.38) can be expressed in the form

$$
f_{1(m+1)}(t)=h_{1(m+1)}(t)-\gamma_{1(m+1)} \int_{0}^{t} h_{1(m+1)}(t-\tau) e^{-\gamma_{1(m+1)} \tau} d \tau,
$$


and so,

$$
\begin{aligned}
\left|f_{1(m+1)}(t)\right| & \leq 2^{m}+2^{m} \gamma_{1(m+1)} \int_{0}^{t} e^{-\gamma_{1(m+1)} \tau} d \tau \\
& =2^{m}-2^{m}\left(e^{-\gamma_{1(m+1)}}-1\right) \leq 2^{m+1} .
\end{aligned}
$$

Thus, (2.37) is fulfilled, which completes the proof of Theorem 2.(m+1). This means that Theorem 2.n is satisfied for all $n \in \mathbb{N}$.

A function $K_{1 n}$ satisfying conditions (1) $-\left(3+n+t_{1 n}\right)$ of Theorem 2.n can be obtained by using the following method:

Example 2.n. If there exist the numbers $a_{1 n 0}, a_{1 n 1}, \ldots, a_{1 n(n+1)}>0$ satisfying conditions $(2)-\left(3+n+t_{1 n}\right)$ of Theorem 2.n, then there exists at least one function $K_{1 n}$ which satisfies condition (1) of Theorem 2.n of the form

$$
K_{1 n}(t)=\sum_{m=0}^{n+1} c_{m} t^{n+1-m}+c_{n+2} e^{-t}
$$

such that

$$
K_{1 n}(0)=a_{1 n 0}, K_{1 n}^{\prime}(0)=a_{1 n 1}, \ldots, K_{1 n}^{(n)}(0)=a_{1 n n}, K_{1 n}^{(n+1)}(0)=a_{1 n(n+1)} .
$$

To show the truth of this fact, we first choose the numbers $a_{1 n 0}, a_{1 n 1}>0$ satisfying conditions (2) and (3) of Theorem 2.n and define the polynomial $P_{1 n i}$ for all $i \in$ $\mathbb{N}_{n+2}$, the number $\gamma_{1 n}$ by

$$
\begin{gathered}
P_{1 n i}(\gamma)=\sum_{k=0}^{i}(-\gamma)^{i-k} a_{1 n(k-1)}\left(a_{1 n(-1)} \equiv 1\right. \text { by convention), } \\
\gamma_{11}=\frac{a_{110}}{3} \text { for } n=1 \text { and } \gamma_{1 n}=\frac{a_{1 n 0}}{n+1} \text { for } n \geq 2 .
\end{gathered}
$$

So, if $n \geq 2$, then it can be clearly seen by the proof of Theorem $2 . n$ that the numbers $a_{1(n-1) 0}$ and $a_{1(n-1) 1}$ defined by

$$
a_{1(n-1) 0}=P_{1 n 1}\left(\gamma_{1 n}\right) \text { and } a_{1(n-1) 1}=P_{1 n 2}\left(\gamma_{1 n}\right)
$$

satisfy conditions (2) and (3) of Theorem 2.(n-1). Furthermore, the numbers $a_{1(n-1) 2}, a_{1(n-1) 3}, \ldots, a_{1(n-1) n}>0$ whenever $n \geq 2$ can also be found by means of the constants $a_{1(n-1) 0}, a_{1(n-1) 1}>0$ as those were obtained in Example 2.(n-1). Thus, for $n \geq 2$, by taking

$$
P_{1 n 3}\left(\gamma_{1 n}\right)=a_{1(n-1) 2}, P_{1 n 4}\left(\gamma_{1 n}\right)=a_{1(n-1) 3}, \ldots, P_{1 n(n+1)}\left(\gamma_{1 n}\right)=a_{1(n-1) n},
$$

the numbers $a_{1 n 2}, a_{1 n 3}, \ldots, a_{1 n n}$ are obtained as

$$
\begin{aligned}
a_{1 n 2} & =a_{1(n-1) 2}+\gamma_{1 n} a_{1(n-1) 1}>0, \\
a_{1 n 3} & =a_{1(n-1) 3}+\gamma_{1 n} a_{1(n-1) 2}>0, \\
& \vdots \\
a_{1 n n} & =a_{1(n-1) n}+\gamma_{1 n} a_{1(n-1)(n-1)}>0 .
\end{aligned}
$$

Besides for $n \geq 1$, since condition $\left(3+n+t_{1 n}\right)$ of Theorem 2.n is

$$
P_{1 n(n+2)}\left(\gamma_{1 n}\right)=-\gamma_{1 n} P_{1 n(n+1)}\left(\gamma_{1 n}\right)+a_{1 n(n+1)} \leq 0,
$$

the number $a_{112}>0$ can be taken as

$$
0<a_{112} \leq \gamma_{11} P_{112}\left(\gamma_{11}\right)
$$


whenever $n=1$, and in the case $n \geq 2$, the number $a_{1 n(n+1)}>0$ can be taken as

$$
0<a_{1 n(n+1)} \leq \gamma_{1 n} P_{1 n(n+1)}\left(\gamma_{1 n}\right)=\gamma_{1 n} a_{1(n-1) n} .
$$

Clearly, the numbers $a_{1 n 0}, a_{1 n 1}, \ldots, a_{1 n(n+1)}>0$ obtained by this way satisfy conditions $(2)-\left(3+n+t_{1 n}\right)$ of Theorem 2.n.

The solution of the system of linear equations (2.46) which is equivalent to

$$
\begin{aligned}
c_{n+1}+c_{n+2} & =a_{1 n 0}, c_{n}-c_{n+2}=a_{1 n 1}, \ldots, n ! c_{1}+(-1)^{n} c_{n+2} \\
& =a_{1 n n},(n+1) ! c_{0}+(-1)^{n+1} c_{n+2}=a_{1 n(n+1)}
\end{aligned}
$$

is obtained as

$$
\begin{aligned}
& \left(c_{0}, c_{1}, \ldots, c_{n+1}, c_{n+2}\right) \\
& \quad=\left(\frac{a_{1 n(n+1)}-(-1)^{n+1} c_{n+2}}{(n+1) !}, \frac{a_{1 n n}-(-1)^{n} c_{n+2}}{n !}, \ldots, a_{1 n 0}-c_{n+2}, c_{n+2}\right),
\end{aligned}
$$

where $c_{n+2}$ is an arbitrary constant satisfying the condition

$$
0 \leq c_{n+2}<\min \left\{a_{1 n 0}, a_{1 n 2}, \ldots, a_{1 n(n-1)}, a_{1 n(n+1)}\right\}
$$

if $n$ is odd and

$$
\max \left\{-a_{1 n 1},-a_{1 n 3}, \ldots,-a_{1 n(n-1)},-a_{1 n(n+1)}\right\}<c_{n+2} \leq 0
$$

if $n$ is even. Thus, every $K_{1 n}$ of the form (2.45) satisfies condition (1) of Theorem 2.n as well.

\section{ACKNOWLEDGMENTS}

The authors would like to express their pleasure to Professor Dr. Feyzi Başar, Inönü Üniversitesi, Eğitim Fakültesi, 44280-Malatya/TURKEY, for his valuable helps and suggestions which improved the presentation of the paper. Also, they wish to express their gratitude to the referee for his/her careful reviewing and report.

\section{REFERENCES}

[1] R. Bellman and K. L. Cooke, Differential - Difference Equations, Academic Press, New York, 1963. MR0147745 (26:5259)

[2] R. Ling, Integral equations of Volterra type, J. Math. Anal. Appl. 64 (1978), 381-397. MR0487317 (58:6964)

[3] R. Ling, Solutions of singular integral equations, Internat. J. Math. \& Math. Sci. 5(1) (1982), 123-131. MR0666499 (83j:45005)

[4] I. Özdemir and Ö. F. Temizer, Expansion of the boundaries of the solutions of the linear Volterra integral equations with convolution kernel, Integral Equations Operator Theory 43 (2002), 466-479. MR1909376 (2003b:45001)

[5] F. G. Tricomi, Integral Equations, Interscience Publishers, Inc., New York, 1957. MR0094665 $(20: 1177)$

InÖNÜ ÜNIVERsitesi, EĞITIM FAKÜLtesi, 44280-Malatya, TURKEY

E-mail address: isozdemir@inonu.edu.tr

Inönü Üniversitesi, EĞitim FakÜltesi, 44280-Malatya, Turkey

E-mail address: oftemizer@inonu.edu.tr 